\title{
Confronting the models of 3:2 quasiperiodic oscillations with the rapid spin of the microquasar GRS 1915+105
}

\author{
G. Török, A. Kotrlová, E. Šrámková, and Z. Stuchlík
}

\begin{abstract}
Institute of Physics, Faculty of Philosophy and Science, Silesian University in Opava, Bezručovo nám. 13, 74601 Opava, Czech Republic

e-mail: terek@volny.cz
\end{abstract}

Received 8 August 2010 / Accepted 5 March 2011

\begin{abstract}
Spectral fitting of the spin $a \equiv c J / G M^{2}$ in the microquasar GRS $1915+105$ estimate values higher than $a=0.98$. However, there are certain doubts about this (nearly) extremal number. Confirming a high value of $a>0.9$ would have significant concequences for the theory of high-frequency quasiperiodic oscillations (HF QPOs). Here we discuss its possible implications assuming several commonly used orbital models of 3:2 HF QPOs. We show that the estimate of $a>0.9$ is almost inconsistent with two hot-spot (relativistic precession and tidal disruption) models and the warped disc resonance model. In contrast, we demonstrate that the epicyclic resonance and discoseismic models assuming the $\mathrm{c}$ - and g-modes are favoured. We extend our discussion to another two microquasars that display the 3:2 HF QPOs. The frequencies of these QPOs scale roughly inversely to the microquasar masses, and the differences in the individual spins, such as $a=0.9$ compared to $a=0.7$, represent a generic problem for most of the discussed geodesic 3:2 QPO models. To explain the observations of all the three microquasars by one unique mechanism, the models would have to accommodate very large non-geodesic corrections.
\end{abstract}

Key words. X-rays: binaries - black hole physics - accretion, accretion disks

\section{Introduction}

In the past few years an impressive amount of work has been done on estimating the black hole spin using the X-ray continuum fitting method (see, e.g., McClintock et al. 2006, 2008; Middleton et al. 2006; Done et al. 2007; Shafee et al. 2008; McClintock et al. 2010, 2011). The obtained results indicate that the spins of the individual sources cover almost the whole range spanning from a non-rotating Schwarzschild black hole to near-extreme Kerr black holes (McClintock 2010; McClintock et al. 2010, 2011). The latter type of object has been detected in the microquasar GRS 1915+105 for which it was estimated by McClintock et al. (2006) that the dimensionless spin $a \equiv$ $c J / G M^{2}$ is higher than $a=0.98$. However, some doubts remain about the (nearly) extremal value reported by McClintock et al. In particular, using a similar fitting procedure Middleton et al. (2006) derived a value of $a=0.7$. Recent independent spin estimates of GRS $1915+105$ based on another, so called, relativistic iron line profile fitting imply the spin either around 0.6 or 0.98 (Blum et al. 2009). There are several concerns about both of the spectral (continuum and iron-line) spin estimate methods. It is not the purpose of our paper to list and analyze these reasons in detail but it is useful to recall some findings of a direct relevance to observations of low-mass X-ray binaries (LMXBs).

Within continuum method studies, the X-ray spectrum is assumed to be the sum of black body contributions from different disc radii. Within the adopted concept, the expected spectrum is determined by the radial distribution of disc temperature, which follows from the relativistic version of the Shakura-Sunyaev accretion disc model and depends on the black hole spin (Shakura \& Sunayev 1973; Novikov \& Thorne 1973). The spin is then inferred by fitting the observed disc spectra to those calculated. In more detail, one of the key theoretical assumptions of this method lies in the expectation that the inner edge of the accretion disc coincides with the innermost stable circular orbit (ISCO). Consequently, the spectral fitting provides an estimate of the ISCO position that depends on the black hole spin (see Shafee et al. 2006; McClintock \& Remillard 2009; Penna et al. 2010, for a more complex picture).

The method deals only with a subset of observations where the assumption of the blackbody emission seems valid. Thus, only datasets that show (presumably) weakly Comptonized spectra acquired for the thermal dominant source state have been used in several works (e.g., McClintock \& Remillard 2003, 2009; Shafee et al. 2008; but see also Steiner et al. 2009). Moreover, only observations where the source luminosity is less than $\sim 20 \%$ of the Eddington luminosity provide reliable results, since at high luminosities the standard ISCO concept is not valid (see Done \& Davis 2008; Abramowicz et al. 2010). The method is also sensitive to the accuracy of the source distance, mass, and inclination determined from independent methods (usually from the optical measurements).

The iron-line method studies are based on the evidence of a broad, skewed iron-line profile that is also observed in the spectra of several LMXBs. The observed iron line is believed to originate from the reflection of hard X-ray photons in the innermost parts of the accretion disk. These photons, emitted by an external process (often assumed to be inverse-Compton scattering in a hot corona), have sufficient energy to remove the $\mathrm{K}$-shell electrons from the iron atoms in the accreted gas and produce the $\mathrm{Fe}-\mathrm{K}$ emission. The broad profile of the $\mathrm{Fe}-\mathrm{K}$ line is then explained by relativistic Doppler effects, light bending, and the 
gravitational redshift characteristic of the vicinity of the binary central compact object (see Miller 2007, for a review). As for spectral continuum fitting, it is assumed that the disc extends down to ISCO. Consequently, properties of the broadened emission line are associated with the ISCO radii when considering the spin as a free parameter. The advantage of this method is that it does not require accurate information about the source distance and mass, and provides estimate of the source inclination itself (e.g., Davis et al. 2006; McClintock \& Remillard 2009). Its weak points are connected namely to important requirements about the strength of the detected signal and to difficulties in subtracting the line from the whole spectra (when adopting detailed modeling of the continuum). Moreover, there are doubts that the line broadening is caused by general relativistic effects, since these strongly depend not only on the radius of the emission, but also on details of the local emission that are not very known (e.g., Beckwith \& Done 2004).

Both of the aforementioned means of estimating the black hole spin rely on being able to relate the source states to the disc geometry. In the case of GRS $1915+105$, it is unclear how this can be achieved (e.g., McClintock \& Remillard 2003; Done et al. 2004; van Oers et al. 2010; Li et al. 2010). In view of all the uncertainties, one should keep in mind that some caution is needed in relation to the high value of the black hole spin in GRS $1915+105^{1}$.

\section{Orbital models of quasiperiodic oscillations}

The X-ray power density spectra (PDS) of several LMXBs obtained during the past three decades contain peaked features called quasi-periodic oscillations (QPOs). These QPOs are observed in neutron star (NS) as well as black hole (BH) systems and occur with periods of the order of $1-10^{-3} \mathrm{~s}$. An outstanding example of these oscillations arises in the high frequency part of the PDS being thus called "high frequency" (HF) QPOs. When more HF QPOs are observed in black hole sources, their frequencies seem to form commensurable pairs with a preferred ratio of 3:2 (Abramowicz \& Kluźniak 2001; McClintock \& Remillard 2003). The black hole HF QPOs are typically weaker and less coherent than most of the NS HF QPOs (e.g., Barret et al. 2005a,b; Méndez 2006). The neutron star HF QPOs appear as two correlated modes at distinct (but, in contrast to $\mathrm{BH}$ HF QPOs, variable) frequencies. The range of frequencies spanned by the two NS modes is rather large (ranging from $50 \mathrm{~Hz}$ to $1500 \mathrm{~Hz}$, being typically a few hundreds of $\mathrm{Hz}$ for each individual source). The two NS modes are in a way analogous to the $\mathrm{BH}$ case because they usually exchange their dominance when passing the 3:2 frequency ratio (Török et al. 2008a,b,c; Török 2009; Boutelier et al. 2010). Properties of the HF QPOs are reviewed in, e.g., McClintock \& Remillard (2003) or van der Klis (2006).

There is strong evidence that the HF QPOs originate very close (less than 100 gravitational radii $r_{\mathrm{g}} \equiv \mathrm{GMc}^{-2}$ ) to the accreting compact objects, but no commonly accepted QPO theory has so far been developed (e.g., van der Klis 2006).

\footnotetext{
${ }^{1}$ McClintock et al. (2011) states that $a>0.98$ but the authors also note that some caution is needed namely because of the incomplete study of the source distance (see their paper for details). In this paper, we show that confirming it would have a significant impact on the theory of the high-frequency quasiperiodic oscillations (HF QPOs) and present the discussion of some concrete implications for a few frequently quoted QPO models.
}

Moreover, while there are several concrete, well-described similarities and differences between the black hole and neutron star HF QPO phenomenology (including those briefly mentioned above), it has not yet been resolved whether the generic mechanism could be the same for both classes of the sources. There are several proposed models that cannot be applied to both the classes, e.g., due to the requirement of a solid NS surface (Lamb et al. 1985; Alpar \& Shaham 1985) or a high black hole spin (Stuchlík et al. 2007a,b; Slaný \& Stuchlík 2008). Nevertheless, a larger variety of models has been designed under the assumption of a common mechanism (see, e.g., van der Klis 2006, for a basic overview). Most of the hypotheses assume a relation between the QPO frequencies and the frequencies related to motion of accreted matter orbiting in the vicinity of a compact object (hereafter "orbital" models). These models typically deal with either "hot-spot" or "disc-oscillation" QPO interpretation. We next consider an arbitrary choice of several commonly quoted models. For the sake of comprehensibility, we first give a short summary of the examined models and recall some of their main features and related references.

\subsection{Kinematic models}

The kinematics of the orbital motion allows the consideration of the variability that arises from the motion of "hot-spots" orbiting inside the accretion disc. We consider two models of this kind. The "relativistic precession" model (hereafter RP model) was proposed in a series of papers by Stella \& Vietri (1998a, 1999, 2002); Morsink \& Stella (1999). The model illustrates that the $\mathrm{kHz}$ QPOs represent modes of the relativistic epicyclic motion of blobs at various radii $r$ in the inner parts of the accretion disc. It is often recalled and known for roughly matching the correlation between the HF QPOs observed in the NS sources (e.g., Belloni et al. 2007a; Török et al. 2010; Lin et al. 2010). Within the model, the twin-peak QPO frequency correlation arises because of the periastron precession of the relativistic orbits. Owing to Lense-Thirring relativistic precession, the model also predicts another frequency correlation extending to higher timescales. The $\mathrm{kHz}$ QPO frequencies are indeed correlated to the low-frequency QPO features observed around $\sim 1-50 \mathrm{~Hz}$ (e.g., Stella \& Vietri 1998b, this correlation is however not being the focus of our present work). On the other hand, there are apparent difficulties with the detailed modeling of the relation between the hot-spot motion and the observed modulation (e.g., Lamb \& Markovic 2000).

Čadež et al. (2008), Kostić et al. (2009), and Germana et al. (2009) introduced a similar concept in which the QPOs are generated by a "tidal disruption" (TD) of large accreting inhomogeneities. Their hydrodynamic simulations signify that blobs orbiting the central compact object can be stretched by tidal forces forming "ring-section" features within the model expected to be responsible for the observed modulation. The model has been proposed for both supermassive and stellar mass black holes. We note that, at least in some cases, the power density spectra simulated using the model closely reproduce those that are observed. However, the concept of "rocks" approaching the radii of the order of ISCO-radius in LMXBs is questionable. The upper limit to the radii, where the tidal forces begin to disrupt the object of density $\rho$ orbiting a primary source of mass $M$, is roughly described by the Roche-limit $r_{\mathrm{TD}} \sim(M / \rho)^{1 / 3}$. The related characteristic orbital frequency $v_{\mathrm{TD}} \sim\left(\mathrm{G} M / r_{\mathrm{TD}}^{3}\right)^{1 / 2}$ can be expressed as 
Table 1. Frequency relations corresponding to individual QPO models and the spins implied by the 3:2 QPOs in GRS $1915+105$ and the massrange $10-18 M_{\odot}$.

\begin{tabular}{llllr}
\hline \hline Model & \multicolumn{2}{c}{ Relations } & $v_{\mathrm{K}} / v_{\mathrm{r}}$ or ${ }^{*} v_{\theta} / v_{\mathrm{r}}$ & $a \sim$ \\
\hline RP & $v_{\mathrm{L}}=v_{\mathrm{K}}-v_{\mathrm{r}}$ & $v_{\mathrm{U}}=v_{\mathrm{K}}$ & $3 / 1^{*}$ & $<0.55$ \\
TD & $v_{\mathrm{L}}=v_{\mathrm{K}}$ & $v_{\mathrm{U}}=v_{\mathrm{K}}+v_{\mathrm{r}}$ & $2 / 1$ & $<0.45$ \\
\hline $\mathrm{WD}$ & $v_{\mathrm{L}}=2\left(v_{\mathrm{K}}-v_{\mathrm{r}}\right)$ & $v_{\mathrm{U}}=2 v_{\mathrm{K}}-v_{\mathrm{r}}$ & $2 / 1$ & $<0.45$ \\
Ep & $v_{\mathrm{L}}=v_{\mathrm{r}}$ & $v_{\mathrm{U}}=v_{\theta}$ & $3 / 2^{*}$ & $0.65-1$ \\
Kp & $v_{\mathrm{L}}=v_{\mathrm{r}}$ & $v_{\mathrm{U}}=v_{\mathrm{K}}$ & $3 / 2^{*}$ & $0.70-1$ \\
$\mathrm{RP} 1$ & $v_{\mathrm{L}}=v_{\mathrm{K}}-v_{\mathrm{r}}$ & $v_{\mathrm{U}}=v_{\theta}$ & - & $<0.80$ \\
$\mathrm{RP} 2$ & $v_{\mathrm{L}}=v_{\mathrm{K}}-v_{\mathrm{r}}$ & $v_{\mathrm{U}}=2 v_{\mathrm{K}}-v_{\theta}$ & - & $<0.45$ \\
\hline
\end{tabular}

Notes. The middle column indicates the ratio of the epicyclic frequencies determining the radii corresponding to the observed 3:2 ratio. The indicated ranges of spin also represent total spin ranges for the whole group of the three microquasars (see Fig. 2).

$v_{\mathrm{TD}}=(\mathrm{G} \rho)^{1 / 2}$. For the rocks it is clearly given by $v_{\mathrm{TD}} \sim 10^{-3} \mathrm{~Hz}$, which is far lower than the observed $(\sim 100 \mathrm{~Hz})$ frequencies ${ }^{2}$.

Identification of the lower and upper $\mathrm{kHz}$ QPO frequencies with the frequencies of the orbital motion is recalled in the top part of Table 1 for both hot spot models. To match the observed 3:2 ratio, the QPOs in the RP model must be generated at the radii where $v_{K} / v_{\mathrm{r}}=3 / 1\left(r=6.75 \mathrm{GMc}^{-2}\right.$ for $\left.a=0\right)$, i.e., very close to ISCO $\left(r=6 \mathrm{GMc}^{-2}\right.$ for $\left.a=0\right)$ where the radial epicyclic frequency vanishes. For the TD model, this location is shifted outwards close to the radius where the radial epicyclic frequency exhibits a maximum value $\left(v_{K} / v_{\mathrm{r}}=2 / 1 ; r=8 \mathrm{GMc}^{-2}\right.$ for $\left.a=0\right)$.

\subsection{Resonant models}

Within the (present) RP model, there is no generic explanation of the observed 3:2 frequency ratio $R=3 / 2$ and there is a clear need to explore the issue of the preference of certain orbits. The concept of the TD model is less problematic because for any $a$ and $r$ it implies that $R \in(1,2)$ and the predicted effects occur around the location of the maximal allowed $v_{\mathrm{r}}$, where $v_{K} / v_{\mathrm{r}} \sim 2 / 1$. Nevertheless, the frequency ratio is also not reliably constrained by this model. In contrast, the commensurability of the frequencies is crucial for the models when assuming the warped disc (WD) oscillations suggested by Kato (2001, 2004a,b, 2005, 2008). Kato (2008) reviewed several possible resonances in deformed disc (see also works of Ferreira $\&$ Ogilvie 2008, 2009). The main weak point of the concrete WD model assumed here (Kato 2004a,b, see Table 1) is that it considers a somewhat exotic disc geometry that causes a doubling of the observed lower QPO frequency.

The frequency commensurability is also crucial for the nonlinear resonance models discussed by Abramowicz, Kluźniak and collaborators (Kluźniak \& Abramowicz 2001; Abramowicz \& Kluźniak 2001; Abramowicz et al. 2003a,b; Bursa et al. 2004; Rebusco 2004; Horák 2008; Stuchlík et al. 2008a,b; Horák et al. 2009, and others; see also Aliev \& Galtsov 1981; and Aliev 2007). A particular resonance of this kind that is often discussed is the 3:2 internal epicyclic or Keplerian resonance (Ep, Kp, see Török et al. 2005, for details). The explicit formulae of the frequency relations corresponding to these three resonant disc oscillation models are listed in the bottom part of Table 1 . We note that while for the WD resonance model the QPOs are located at the same radii as for the kinematic TD model, i.e., around the location of the maximum of $v_{\mathrm{r}}$, the two $3: 2$ resonances occur

\footnotetext{
${ }^{2}$ We thank the anonymous referee for emphasizing this issue.
}

above this maximum, at a larger distance from the central object. We also consider here another two QPO (resonance) models that we denote as RP1 model (Bursa 2005) and RP2 model (Török et al. 2010). Both of them assume different combinations of non-axisymmetric disc-oscillation modes. They are of particular interest because they involve oscillation modes whose frequencies for slow rotation almost coincide with the frequencies predicted by the RP model. These two resonances should occur much closer to ISCO than the resonance expected in the Ep model.

The physical interpretation of the RP1 model is unclear, since the oscillation modes assumed within the model are unlikely to be able to enter the resonance (see, Horák 2008, for details). The resonant coupling between the pairs of the oscillation modes assumed in the Kp, EP, and RP2 models is in principle allowed, but detailed physical mechanisms providing this coupling and mode excitations have not yet been fully developed (see, e.g., Šrámková et al. 2007; Horák 2008; Rebusco 2008; Kluźniak 2008, and references therein). Substantial effort clearly yet needs to be invested in extending both the related analytic and numerical work and the link between them.

\subsection{Models assuming fundamental discoseismic modes}

We have so far only considered models in which it is assumed that both of the observed 3:2 frequencies are produced by the same mechanism and excited at a certain (common) preferred radius. A qualitatively different consideration relating each of the two frequencies to a different radial region arises when general combinations of the fundamental discoseismic modes are assumed. The HF QPOs have been proposed to correspond to the following three distinct modes: the so-called g-modes (inertialgravity waves that occur at the radius where the radial epicyclic frequency reaches its maximum value), c-modes (corrugation vertically incompressible waves near the inner edge of the disk), and p-modes (inertial-pressure oscillations that occur near the edge of the disc). This concept was elaborated in particular by Kato \& Fukue (1980), Okazaki et al. (1987), Nowak \& Wagoner (1992), Wagoner (1999), Silbergleit et al. (2001), Wagoner et al. (2001), Ortega-Rodríguez et al. (2002), Silbergleit \& Wagoner (2008), and Wagoner (2008). Disc oscillation modes that have been proposed to explain the QPOs have been observed in hydrodynamical (HD) simulations of the accretion processes (e.g., Zanotti et al. 2005; Reynolds \& Coleman 2009) but there is growing evidence from magnetohydrodynamic (MHD) simulations that these modes are typicaly dumped by various instabilities produced in the presence of the magnetic field (see, e.g., Tsang \& Lai 2009; Fu \& Lai 2009, 2011). At the same time, the MHD simulations do not convincingly reproduce the 3:2 QPOs that undoubtedly appear in the X-ray fluxes of the LMXBs. It is therefore also unclear whether these simulations accomodate all the crucial ingredients or not (see, e.g., Machida \& Matsumoto 2007, 2008, who found in their MHD simulations some low frequency QPOs only in the presence of the radiative cooling).

\subsection{Confronting the QPO models with the rapid spin of black hole in the microquasar GRS 1915+105}

In general, the existing QPO models represent rather unfinished concepts that have specific advantages and difficulties. The oscillations predicted by these models are usually not seen in the present MHD simulations. Moreover, none of these models 

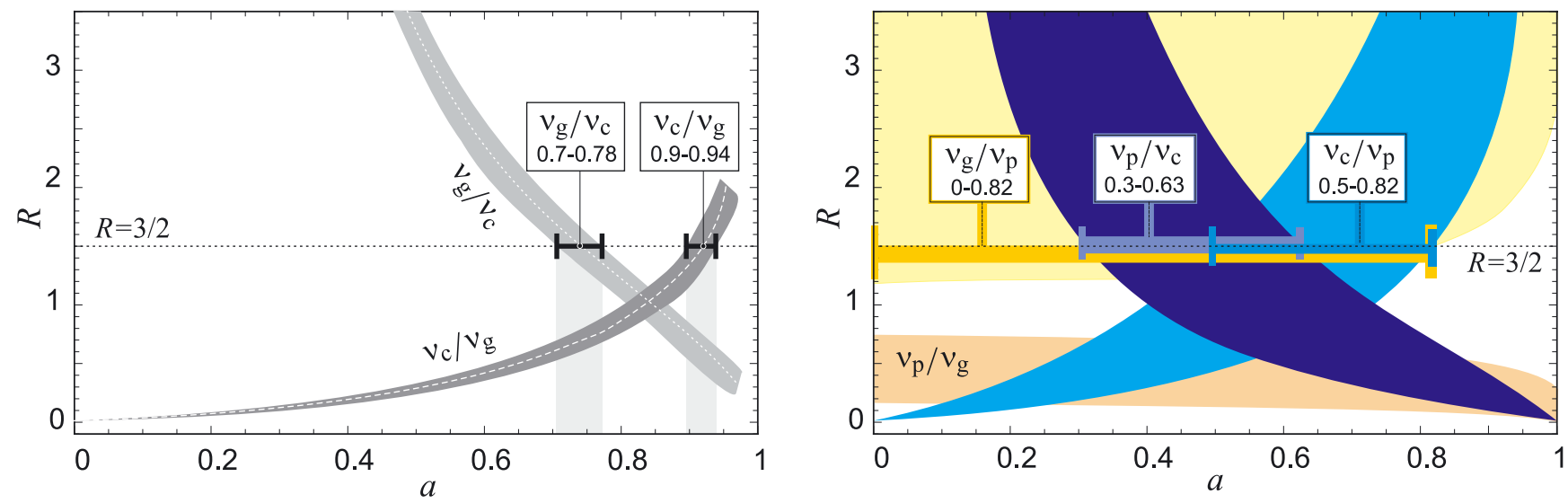

Fig. 1. Ratio of the frequencies of the fundamental discoseismic modes (based on Wagoner et al. 2001). Left: ratio of $v_{\mathrm{c}}$ to $v_{\mathrm{g}}$. The spread of two functions corresponds to the uncertainty in the speed of sound. The horizontal dotted line denotes the 3:2 frequency ratio. Values in the boxes evaluate the spin ranges required by this ratio. Right: the same consideration, but including the p-modes.

yet really match the full QPO phenomenology observed in the LMXBs including the dependence of the QPO visibility on the source spectral states, QPO amplitudes, and coherence times. In this situation, we expect that the implications of the eventually confirmed high spin of GRS 1915+105 could help us improve the individual QPO models or even discriminate between them. In the next section, we calculate the spin implied by the above-mentioned QPO models.

\section{The spin implied by individual models}

There is a straightforward connection between the hot-spot models and the characteristic orbital frequencies (i.e., the three frequencies of the perturbed circular orbital motion: the azimuthal "Keplerian" frequency, the radial epicyclic frequency, and the vertical epicyclic frequency). The frequencies of numerous discoscillation modes are also constrained by the three characteristic orbital frequencies. Assuming a Kerr geometry, the orbital frequencies for a given radius depend only on mass and spin of the black hole ${ }^{3}$. It is therefore possible to infer the black hole spin or mass from the observed 3:2 frequencies and (at least some) concrete orbital models. This procedure has been previously performed, e.g., for a wide selection of the resonance QPO models (Abramowicz \& Kluźniak 2001; Török et al. 2005; Török 2005). We recall that the complete set of formulae required to evaluate the Keplerian and epicyclic orbital frequencies in Kerr geometry was first derived by Aliev \& Galtsov (1981). These frequencies were extensively discussed in several later studies. A detailed analysis can be found in Török \& Stuchlík (2005), where the definition formulae are given in the usual form and the frequencies are studied across the full range of positive $a$. We note that here we do not consider $a>1$ since we focus our attention fully on models originally designed for BHs and not on their possible extensions to naked singularities.

The 3:2 QPO frequencies in GRS 1915+105 are well known to be given by (McClintock \& Remillard 2003)

$v_{\mathrm{U}}=168 \pm 3 \mathrm{~Hz}$ and $v_{\mathrm{L}}=113 \pm 5 \mathrm{~Hz}$

Assuming Eq. (1) and the aforementioned formulae for the orbital frequencies, we calculate the implied mass-spin functions

\footnotetext{
${ }^{3}$ We consider here only the Kerr spacetime as a standard description for rotating BHs, although alternatives have been discussed in a similar context (see Kotrlová et al. 2008; Stuchlík \& Kotrlová 2009).
}

for the models associating the 3:2 QPOs with a common radii by means of the definition relations given in Table 1 . Following Abramowicz \& Kluźniak (2001) and Török et al. (2005) and taking into account the estimated range of the mass of GRS 1915+105 (e.g., McClintock \& Remillard 2003)

$10 M_{\odot} \leq M \leq 18 M_{\odot}$,

we infer the expected ranges of the spin. We found that the RP, TD, WD and RP2 models imply that the spin is rather too low, i.e. $a<0.6$. Somewhat more satisfactory are the predictions obtained from the $\operatorname{Ep}(0.65 \leq a \leq 1), \operatorname{Kep}(0.7 \leq a \leq 1)$, and RP1 $(a \leq 0.8)$ models. For each model, the detailed results are presented in the last column of Table 1. As recalled above, for the discoseismic modes the individual observed QPOs correspond to different modes located at their own radii. The frequencies of these modes depend on the black hole spin and the speed of sound in the accreted gas, and scale roughly as $1 / M$, whereas their dependence on the other parameters of the accreting system is supposed to be very weak (Wagoner et al. 2001). The frequencies of the c- and g-modes $\left(v_{\mathrm{c}}\right.$ and $\left.v_{\mathrm{g}}\right)$ depend only moderately on the speed of sound. Thus, their ratio is mainly a function of the black hole spin, which is depicted in the left panel of Fig. 1. The right panel of Fig. 1 shows the relations of the ratios of the frequencies $v_{\mathrm{c}}$ or $v_{\mathrm{g}}$ to the p-mode frequency $v_{\mathrm{p}}$, which are strongly dependent on the speed of sound. Inspecting both panels of Fig. 1, we can see that for the combination of modes relating $v_{\mathrm{U}}=v_{\mathrm{p}}$ and $v_{L}=v_{\mathrm{g}}$ the frequency ratio $R$ is never higher than unity. For the other five possible combinations of modes, there is in each case some range of $a$ that corresponds to the 3:2 frequency ratio. This range is located above $a=0.9$ only for the combination relating $v_{\mathrm{U}}=v_{\mathrm{c}}$ and $v_{L}=v_{\mathrm{g}}$. In more detail, this combination requires that $0.9 \leq a \leq 0.94^{4}$. The mass needed to match $v_{\mathrm{U}}=168 \pm 3 \mathrm{~Hz}$ is then $13 M_{\odot} \leq M \leq 19 M_{\odot}$ with good overlap $13 M_{\odot} \leq M \leq 18 M_{\odot}$ with the expected mass given in Eq. (2).

4 For higher values of $a$, the model predictions seem to deviate from the $3: 2$ ratio since the c-mode frequencies, when $a$ increases, grow faster than the frequencies of the g-mode. However, the published works have not fully investigated the behaviour for very high spin values $a>0.95$ so far because of numerical difficulties (Wagoner 2010, from a private communication). 


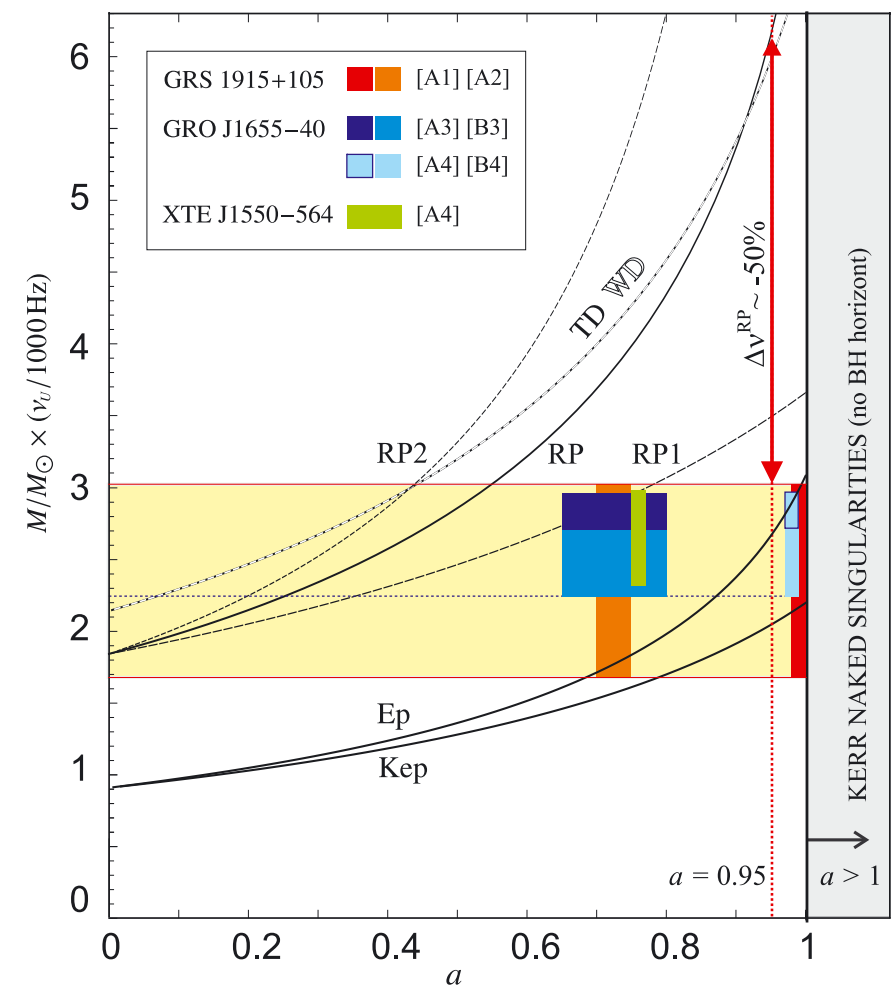

Fig. 2. Curves $v_{\mathrm{U}} \times M(a)$ implied by the individual geodesic models. The light yellow rectangle indicates the observationaly determined interval of $v_{\mathrm{U}} \times M$ for GRS $1915+105$. The red dotted vertical line denotes $a=0.95$. The red vertical arrow indicates the correction needed to match the upper limit to $v_{\mathrm{U}} \times M$ with the RP model for this spin. The colour boxes are drawn for the mass and spectral spin estimates given by different authors for GRS 1915+105, GRO J1655-40 and XTE 1550-564 (see the main text for references). The dotted blue line indicates the lower observational limit to $M \times v_{\mathrm{U}}$ that is roughly common to GRO J1655-405 and XTE 1550-564.

\section{Discussion and conclusions}

It follows from our previous discussion that the hypothesis of the internal (epicyclic) resonance 5 and the discoseismic model which relates the upper and lower 3:2 QPO to the c- and g-modes are favoured in the case of GRS $1915+105$ provided that $a>0.9$. The TD, WD, RP, and RP2 models are then disfavoured. This statement was inferred assuming that $v_{K}, v_{\mathrm{r}}$, and $v_{\theta}$ are the exact geodesic frequencies. A similar analysis including the influence of non-geodesic effects would require very detailed study. Here we only roughly estimate the possible relevance of the nongeodesic effects. Figure 2 shows the $v_{\mathrm{U}} \times M(a)$ curves implied by the individual geodesic models. These curves (as well as the curves drawn in the consequent Fig. 3) have non-trivial ambiguous extensions for the range of $a>1$ representing the nakedsingularity region. We do not consider them here, since we focus our attention on black holes (we expect to discuss the subject of $a>1$ in a different work). The observationally determined interval of $v_{U} \times M$ for GRS $1915+105$ is indicated by the light yellow rectangle. We define the relative non-geodesic correction to be

$$
\Delta v \equiv\left(v_{\text {observed }}-v_{\text {predicted }}\right) / v_{\text {predicted }}
$$

\footnotetext{
5 Török et al. (2005) assumed this and several other resonances for the spin estimates of the Galactic microquasars. From the other considered resonances, the " $5: 1$ " resonance can also match the high spin. This resonance involves relatively high resonant coefficients and occurs very close to ISCO.
}

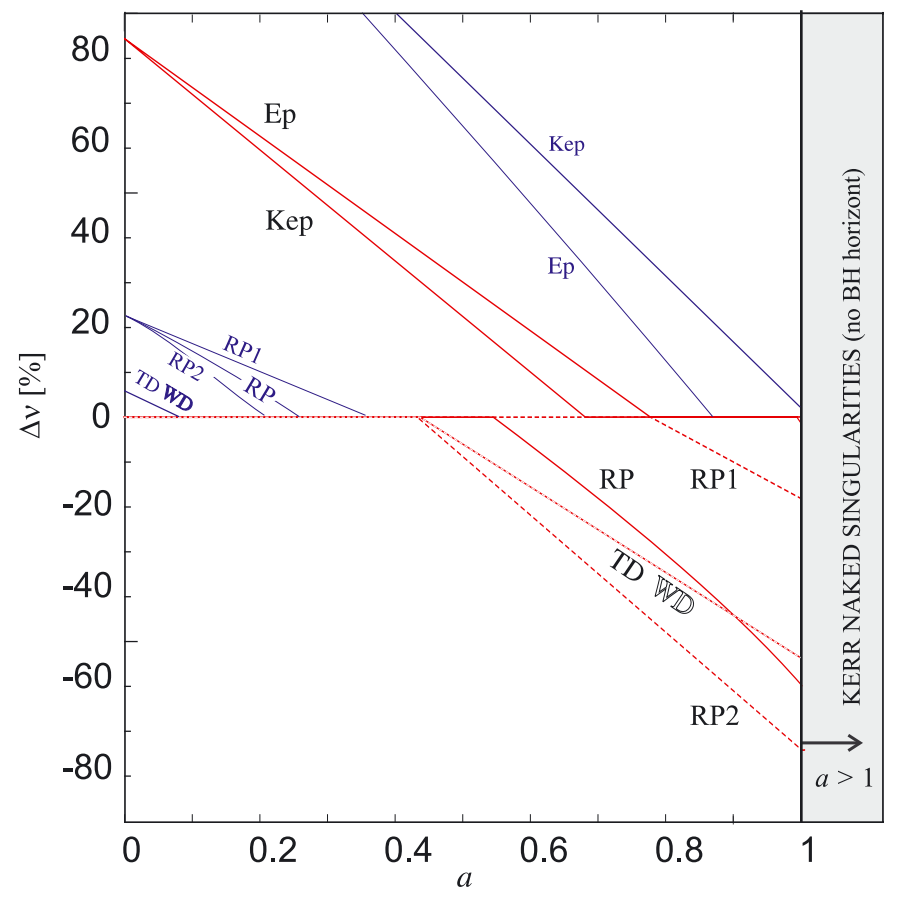

Fig. 3. Non-geodesic corrections required for a given model, spin and source. The red curves indicate the minimal corrections required in the case of GRS 1915+105. Parts of these curves with a negative sign also roughly indicate the corrections required for the other two microquasars. The blue curves indicate positive corrections for these microquasars determined by the lower limit to their $M \times v_{\mathrm{U}}$ indicated by the blue dotted horizontal line in Fig. 2.

which is needed to match the observations of GRS $1915+105$ with a given model for a certain spin. From Fig. 2, we can easily estimate by eye that the non-geodesic correction $\Delta v^{\mathrm{RP}}$ required for the frequency predicted by the RP model for $a=0.95$ is about $-50 \%$. From Fig. 3, we can find that for $a \in(0.9,1)$, the quantity $\Delta v^{\mathrm{RP}}$ changes from $-40 \%$ to $-60 \%$. The same is roughly true for the TD and WD models, while for the RP2 model the required correction is even higher. Thus, our result is justified, except when considering of very large nongeodesic corrections.

\subsection{Relation to other sources}

Török et al. (2005) pointed out that since the 3:2 QPO frequencies in microquasars scale roughly as $v_{\mathrm{U}} \doteq 2.8\left(M / M_{\odot}\right)^{-1} \mathrm{kHz}$ (McClintock \& Remillard 2003), their spins implied by the epicyclic resonance model should not vary much among them. In Fig. 2, we include several examples of spin estimates obtained by different groups and methods for GRS $1915+105$ and the other two microquasars, GRO J1655-40 and XTE 1550-564. The references related to these estimates are as follows (the assumed ranges of $v_{\mathrm{U}}$ being taken from McClintock \& Remillard 2003). For the mass, Greene et al. (2001), Greiner et al. (2001), Orosz et al. (2002), Beer \& Podsiadlowski (2002), and McClintock \& Remillard (2003) provide commonly accepted mass estimates. These are in Fig. 2 denoted by letter A. Beer \& Podsiadlowski (2002) present an alternative prediction (in Fig. 2 denoted by letter B) that moves the lower boundary of estimated mass of GRO J1655-40 from 6.0 to $5.1 M_{\odot}$. For the spin, there were the following studies: [1] McClintock et al. (2006); [2] Middleton et al. (2006); [3] McClintock et al. (2008); [4] Miller et al. (2009). 
A\&A 531, A59 (2011)

Table 2. Ranges of $a$ and $M$ implied by the discoseismic models of the 3:2 QPOs.

\begin{tabular}{ccccc}
\hline \hline & & GRS 1915+105 & XTE J1550-564 & GRO J1655-40 \\
Frequencies & $a$ & $M / M_{\odot}[10.0-18.0]$ & $M / M_{\odot}[8.4-10.8]$ & $M / M_{\odot}[5.1-6.6]$ \\
\hline$v_{\mathrm{U}}=v_{\mathrm{g}}, v_{L}=v_{\mathrm{c}}$ & $0.70-0.78$ & $6.4-9.0$ & $3.9-5.5$ & $2.4-3.3$ \\
$v_{\mathrm{U}}=v_{\mathrm{c}}, v_{L}=v_{\mathrm{g}}$ & $0.90-0.94$ & $12.8-19.1$ & $7.8-11.6$ & $4.8-7.1$ \\
\hline$v_{\mathrm{U}}=v_{\mathrm{p}}, v_{L}=v_{\mathrm{c}}$ & $0.30-0.63$ & $1.1-5.0$ & $0.7-3.0$ & $0.4-1.8$ \\
$v_{\mathrm{U}}=v_{\mathrm{c}}, v_{L}=v_{\mathrm{p}}$ & $0.50-0.82$ & $1.7-8.6$ & $1.0-5.2$ & $0.6-3.2$ \\
\hline$v_{\mathrm{U}}=v_{\mathrm{g}}, v_{L}=v_{\mathrm{p}}$ & $0.00-0.82$ & $3.9-9.7$ & $2.4-5.8$ & $1.5-3.6$ \\
$v_{\mathrm{U}}=v_{\mathrm{p}}, v_{L}=v_{\mathrm{g}}$ & - & - & - & - \\
\hline
\end{tabular}

Notes. The values in the brackets indicate the referential interval of $M$ for each microquasar.

The above quoted spin estimates assume either the spectral continuum or the iron line method. It is apparent from Fig. 2 that both different authors and different methods suggest (somewhat) different values of spin (and for one microquasar even different values of mass). The epicyclic resonance model favoured (along with the discoseismic model) in the GRS 1915+105 seems to match at least some of these estimates, but for the parameters of the XTE J1550-564 $\left(a \sim 0.7, M \sim 5-7 M_{\odot}\right)$ assumed in the figure it fails. If different spins $(a>0.9$ in GRS $1915+105$ and $a \sim 0.7$ in GRO J1655-40 and XTE J1550-564) were confirmed, the difficulty of matching the all observed 3:2 frequencies would clearly be rather generic for most of the orbital QPO models.

The observationally determined ranges of $v_{\mathrm{U}} \times M$ for GRO J1655-40 and XTE J1550-564 nearly coincide, the upper limits being roughly equal to the upper limit for GRS $1915+105$ (see Fig. 2). Taking advantage of this setup, we plot a simple rough scheme of the non-geodesic corrections required for a given model, spin, and source in Fig. 3. The red curves in the figure indicate the minimal corrections required for GRS $1915+105$. Parts of these curves with a negative sign also roughly indicate the corrections required for the other two microquasars. Positive corrections for these microquasars are then included as the blue curves. If the values $a>0.9$ for GRS $1915+105$ and $a \sim 0.7$ for GRO J1655-40 or XTE J1550564 were confirmed, the requested corrections would appear to be rather high for most of the denoted models assuming a unified 3:2 QPO mechanism. Only the RP1 model can survive with corrections of $|\Delta v|$ up to $\sim 20 \%$ (but as recalled in Sect. 2, the present physical interpretation of this model is questionable).

Because of the observational $1 / M$ scaling, the above difficulty also arises for the discoseismic models (which are not considered in Figs. 2 and 3). For these, we present in Table 2 the mass ranges implied by all combinations of the fundamental modes. These appear to overlap well with those observationally determined only for the model relating the upper and lower 3:2 QPO to the c- and g-mode provided that $a \in(0.90-0.94)$. For the other combinations related to different spins, the mass ranges differ from those in the observation. Clearly, there is the need for a substantial correction also for a unified 3:2 QPO model assuming fundamental discoseismic modes provided that microquasars had different spins of $a>0.9$ compared to $a \sim 0.7$.

Acknowledgements. We thank M. Abramowicz, W. Kluźniak and R. Wagoner for their comments and the anonymous referee for objections and suggestions that helped to greatly improve the paper. We thank to J. McClintock and Horák, J. for discussion. The authors would also like to acknowledge the support of the Czech grants MSM 478130590, GAČR 202/09/0772 and LC 06014, and the internal grants of SU Opava, FPF SGS/1,2/2010. Part of the work reported here was carried out during the stay of G.T. and Z.S. at the University of Gothenburg which was co-supported by The Swedish Research Council grant (VR) to M. Abramowicz.

\section{References}

Abramowicz, M. A., \& Kluźniak, W. 2001, A\&A, 374, L19

Abramowicz, M. A., Bulik, T., Bursa, M., \& Kluźniak, W. 2003a, A\&A, 404, L21

Abramowicz, M. A., Karas, V., Kluźniak, W., Lee, W. H., \& Rebusco, P. 2003b, PASJ, 55, 466

Abramowicz, M. A., Jaroszynski, M., Kato, S., et al. 2010, A\&A, 521, A15

Aliev, A. N. 2007, Proceedings of the Eleventh Marcel Grossmann Meeting (Singapore: World Scientific)

Aliev, A. N., \& Galtsov, D. V. 1981, GReGr, 13, 899

Alpar, M. A., \& Shaham, J. 1985, Nature, 316, 239

Barret, D., Olive, J. F., \& Miller, M. C. 2005a, MNRAS, 361, 855

Barret, D., Olive, J. F., \& Miller, M. C. 2005b, AN, 326, 808

Beckwith, K., \& Done, C. 2004, MNRAS, 352, 353

Beer, M. E., \& Podsiadlowski, P. 2002, MNRAS, 331, 351

Belloni, T., Méndez, M., \& Homan, J. 2007, MNRAS, 376, 1133

Blaes, O. M., Šrámková, E., Abramowicz, M. A., Kluźniak, W., \& Torkelsson, U. 2007, ApJ, 665, 653

Blum, J. L., Miller, J. M., Fabian, A. C., et al. 2009, ApJ, 706, 66

Boutelier, M., Barret, D., Lin, Y., \& Török, G. 2010, MNRAS, 401, 1290

Bursa, M. 2005, In Proceedings of RAGtime 6/7, Opava

Bursa, M., Abramowicz, M. A., Karas, V., \& Kluźniak, W. 2004, ApJ, 617, L45

Čadež, A., Calvani, M., \& Kostić, U. 2008, A\&A, 487, 527

Davis, S. W., Done, C., \& Blaes, O. M. 2006, ApJ, 647, 525

Done, C., \& Davis, S. W. 2008, ApJ, 683, 389

Done, C., Wardziński, G., \& Gierliński, M. 2004, MNRAS, 349, 393

Done, C., Gierliński, M., \& Kubota, A. 2007, A\&AR, 15, 1

Ferreira, B. T., \& Ogilvie, G. I. 2008, Cool Discs, Hot Flows: The Varying Faces of Accreting Compact Objects, AIP Conf. Proc., 1054, 111

Ferreira, B. T., \& Ogilvie, G. I. 2009, MNRAS, 392, 428

Fu, W., \& Lai, D. 2009, ApJ, 690, 1386

Fu, W., \& Lai, D. 2011, MNRAS, 410, 399

Germana, C., Kostič, U., Čadež, A., \& Calvani, M. 2009, in Simbol-X: Focusing On The Hard X-Ray Universe: Proc. of the 2nd Int. Simbol-X Symp., AIP Conf. Proc., 1126, 367

Greene, J., Bailyn, Ch. D., \& Orosz, J. A. 2001, ApJ, 554, 1290

Greiner, J., Cuby, J. G., \& McCaughrean, M. J. 2001, Nature, 414, 522

Horák, J. 2008, A\&A, 486, 1

Horák, J., Abramowicz, M. A., Kluźniak, W., Rebusco, P., \& Török, G. 2009, A\&A, 499, 540

Kato, S. 2001, PASJ, 53, 1

Kato, S. 2004a, PASJ, 56, 559

Kato, S. 2004b, PASJ, 56, 905

Kato, S. 2005, PASJ, 57, 699

Kato, S. 2008, PASJ, 60, 111

Kato, S., \& Fukue, J. 1980, PASJ, 32, 377

Kluźniak, W. 2008, NewAR, 51, 841

Kluźniak, W., \& Abramowicz, M. A. 2001, unpublished [arXiv: astro-ph/0105057]

Kostić, U., Čadež, A., Calvani, M., \& Gomboc, A. 2009, A\&A, 496, 307

Kotrlová, A., Stuchlík, Z., \& Török, G. 2008, CQGra, 25, 225016

Lamb, F. K., \& Markovic, D. 2000, unpublished [arXiv:astro-ph/0009169]

Lamb, F. K., Shibazaki, N., Alpar, M. A., \& Shaham, J. 1985, Nature, 317, 681

Li, G. X., Yuan, Y. F., \& Cao, X. 2010, ApJ, 715, 623

Lin, Y. F., Boutelier, M., Barret, D., \& Zhang, S. N. 2011, ApJ, 726, 74

Machida, M., \& Matsumoto, R. 2007, in Black Holes from Stars to Galaxies Across the Range of Masses, ed. V. Karas, \& G. Matt, (Cambridge, UK: Cambridge University Press), Proc. IAU Symp., 238, 405

Machida, M., \& Matsumoto, R. 2008, PASJ, 60, 613

McClintock, J. E. 2010, in Conf. on Probing Strong Gravity near Black Holes, held 15-18 February 2010 in Prague 
McClintock, J. E., \& Remillard, R. A. 2003 [arXiv: astro-ph/0306213]

McClintock, J. E., \& Remillard, R. A. 2009, Astro2010: The A\&A Decadal Survey, Science White Papers, No. 197

McClintock, J. E., Shafee, R., Narayan, R., et al. 2006, ApJ, 652, 518

McClintock, J. E., Narayan, R., \& Shafee, R. 2008, In Black Holes, ed. M. Livio, \& A. Koekemoer (Cambridge University Press)

McClintock, J. E., Narayan, R., Gou, L., et al. 2010, Proc. of the Int. Conf. X-ray Astronomy 2009, Present Status, Multi-Wavelength Approach and Future Perspectives, AIP Conf. Proc., 1248, 101

McClintock, J. E., Narayan, R. D., Shane W., et al. 2011, in CQGra, Proc. GR19, ed. D. Marolf, \& D. Sudarsky, accepted [arXiv: 1101.0811]

Méndez, M. 2006, MNRAS, 371, 1925

Middleton, M., Done, Ch., Gierlinski, M., \& Shane, D. W. 2006, MNRAS, 373, 1004

Morsink, S. M., \& Stella, L. 1999, ApJ, 513, 827

Miller, J. M. 2007, ARA\&A, 45, 441

Miller, J. M., Reynolds, C. S., Fabian, A. C., Miniutti, G., \& Gallo, L. C. 2009, ApJ, 697, 900

Nowak, M. A., \& Wagoner, R. V. 1992, ApJ, 393, 697

Novikov, I. D., \& Thorne, K. S. 1973, Black Holes, 343

Orosz, J. A., Groot, P. J., van der Klis, M., et al. 2002, ApJ, 568, 845

Ortega-Rodríguez, M., Silbergleit, A. S., \& Wagoner, R. V. 2002, ApJ, 567, 1043

Okazaki, A. T., Kato, S., \& Fukue, J. 1987, PASJ, 39, 457

Penna, R. F., McKinney, J. C., Narayan, R., et al. 2010, MNRAS, accepted [arXiv: 1003.0966]

Rebusco, P. 2004, PASJ, 56, 553

Rebusco, P. 2008, NewAR, 51, 855

Reynolds, C. S., \& Coleman, C. M. 2009, ApJ, 692, 869

Shafee, R., McClintock, J. E., Narayan, R., et al. 2006, ApJ, 636, L113

Shafee, R., McKinney, J. C., Narayan, R., et al. 2008, ApJ, 687, L25

Shakura, N. I., \& Sunyaev, R. A. 1973, A\&A, 24, 337

Silbergleit, A. S., \& Wagoner, R. V. 2008, ApJ, 680, 1319

Silbergleit, A. S., Wagoner, R. V., \& Ortega-Rodríguez, M. 2001, ApJ, 548, 335

Slaný, P., \& Stuchlík, Z. 2008, A\&A, 492, 319

Šrámková, E. 2005, AN, 326, 9

Šrámková, E., Torkelsson, U., \& Abramowicz, M. A. 2007, A\&A, 467, 641
Steiner, J. F., McClintock, J. E., Remillard, R. A., Narayan, R., \& Gou, L. 2009, ApJ, 701, L83

Stella, L., \& Vietri, M. 1998a, in Abstracts of the 19th Texas Symp. on Relativistic Astrophysics and Cosmology, ed. J. Paul, T. Montmerle \& E. Aubourg (CEA Saclay)

Stella, L., \& Vietri, M. 1998b, ApJ, 492, L59

Stella, L., \& Vietri, M. 1999, Phys. Rev. Lett., 82, 17

Stella, L., \& Vietri, M. 2002, in Proc. of the MGIXMM Meet., 2-8 July 2000, ed. V. G. Gurzadyan, R. T. Jantzen, \& R. Ruffini (World Scientific Publishing), Part A, 426

Straub, O., \& Srámková, E. 2009, CQGra, 26, 055011

Stuchlík, Z., \& Kotrlová, A. 2009, GReGr, 41, 1305

Stuchlík, Z., Slaný, P., \& Török, G. 2007a, A\&A, 463, 807

Stuchlík, Z., Slaný, P., \& Török, G. 2007b, A\&A, 470, 401

Stuchlík, Z., Konar, S., Miller, J. C., \& Hledík, S. 2008a, A\&A, 489, 963

Stuchlík, Z., Kotrlová, A., \& Török, G. 2008b, AcA, 58, 441

Tsang, D., \& Lai, D. 2009, MNRAS, 393, 992

Török, G. 2005, A\&A, 440, 1

Török, G. 2009, A\&A, 497, 661

Török, G., \& Stuchlík, Z. 2005, A\&A, 437, 775

Török, G., Abramowicz, M. A., Kluźniak, W., \& Stuchlík, Z. 2005, A\&A, 436, 1

Török, G., Stuchlík, Z., \& Bakala, P. 2007, CEJP, 5, 457

Török, G., Bakala, P., Stuchlík, Z., \& Čech, P. 2008a, AcA, 58/1, 1

Török, G., Abramowicz, M. A., Bakala, P., et al. 2008b, AcA, 58/1, 15

Török, G., Abramowicz, M. A., Bakala, P., et al. 2008c, AcA, 58/2, 113

Török, G., Bakala, P., Šrámková, E., Stuchlík, Z., \& Urbanec, M. 2010, ApJ, 714,748

van Oers, P., Markoff, S., Rahoui, F., et al. 2010, MNRAS, 409, 763

van der Klis, M. 2006, in Compact Stellar X-Ray Sources, ed. W. H. G. Lewin, \& M. van der Klis (Cambridge University Press), 39

Vio, R., Rebusco, P., Andreani, P., Madsen, H., \& Overgaard, R. V. 2006, A\&A, 452,383

Wagoner, R. V. 1999, Phys. Rev., 311, 259

Wagoner, R. V. 2008, New Astron. Rev., 51, 828

Wagoner, R. V., Silbergleit, A. S., \& Ortega-Rodríguez, M. 2001, ApJ, 559, L25

Zanotti, O., Font, J. A., Rezzolla, L., \& Montero, P. J. 2005, MNRAS, 356, 1371 